\title{
Aortic hemodynamics and white matter hyperintensities in normotensive postmenopausal women
}

\author{
Jill N. Barnes ${ }^{1,2} \cdot$ Ronée E. Harvey $^{1} \cdot$ Samantha M. Zuk $^{3} \cdot$ Emily S. Lundt $^{4}$. \\ Timothy G. Lesnick ${ }^{4}$ Jeffrey L. Gunter ${ }^{5}$ Matthew L. Senjem ${ }^{5} \cdot$ Lynne T. Shuster $^{6}$. \\ Virginia M. Miller ${ }^{2,7} \cdot$ Clifford R. Jack Jr. ${ }^{3} \cdot$ Michael J. Joyner $^{1} \cdot$ Kejal Kantarci $^{3}$
}

Received: 31 January 2017/Revised: 13 March 2017/Accepted: 27 March 2017 /Published online: 7 April 2017

(C) The Author(s) 2017. This article is an open access publication

\begin{abstract}
Hypertension is associated with development of white matter hyperintensities (WMH) in the brain, which are risk factors for mild cognitive impairment. Hormonal shifts at menopause alter vascular function putting women at risk for both hypertension and WMH. Elevations in aortic hemodynamics precede the appearance of clinically defined hypertension but the relationship of aortic hemodynamics to development of WMH in women is not known. Therefore, this study aimed to characterize aortic hemodynamics in relationship to WMH in postmenopausal women. Aortic systolic and diastolic blood
\end{abstract}

Emily Lundt and Timothy Lesnick conducted the statistical analysis and are listed as coauthors.

Kejal Kantarci

Kantarci.Kejal@mayo.edu

Jill N. Barnes

jnbarnes@wisc.edu

Ronée E. Harvey

Harvey.Ronee@mayo.edu

Samantha M. Zuk

Zuk.Samantha@mayo.edu

Emily S. Lundt

Lundt.Emily@mayo.edu

Timothy G. Lesnick

lesnick@mayo.edu

Jeffrey L. Gunter

Gunter.Jeffrey@mayo.edu

Matthew L. Senjem

Senjem.Matthew1@mayo.edu

Lynne T. Shuster

1shuster@mayo.edu

Virginia M. Miller

miller.virginia@mayo.edu pressure (BP), aortic augmentation index (Alx) and aortic round trip travel time (Aortic $T_{\mathrm{R}}$ ) by tonometry were examined in 53 postmenopausal women (age $60 \pm 2$ years). WMH was calculated from fluid-attenuated inversion recovery MRI using a semi-automated segmentation algorithm. WMH as a fraction of total white matter volume positively associated with aortic systolic BP (regression coefficient $=0.018 ; p=0.04$ ) after adjusting for age. In addition, WMH fraction was positively associated with AIx $(0.025 ; p=0.04)$, and inversely associated with Aortic $T_{\mathrm{R}}(-0.015 ; p=0.04)$ after adjusting for age. Our results suggest that assessing aortic hemodynamics may identify individuals at risk for accelerated development of WMH and guide early

Clifford R. Jack Jr.

Jack.Clifford@mayo.edu

Michael J. Joyner

joyner.michael@mayo.edu

1 Department of Anesthesiology, Mayo Clinic, Rochester, MN, USA

2 Department of Physiology and Biomedical Engineering, Mayo Clinic, Rochester, MN, USA

3 Department of Radiology, Mayo Clinic, 200 1st Street SW, Rochester, MN 55905, USA

4 Department of Health Sciences Research, Mayo Clinic, Rochester, MN, USA

5 Department of Informational Technology, Mayo Clinic, Rochester, MN, USA

6 Department of Internal Medicine, Women's Health Clinic, Mayo Clinic, Rochester, MN, USA

7 Department of Surgery, Mayo Clinic, Rochester, MN, USA 
treatment to reduce WMH burden and cognitive impairment in the future.

Keywords MRI - Blood pressure - Cognitive aging · Stroke prevention - Cerebrovascular disease

\section{Introduction}

White matter hyperintensities (WMH) in the brain are risk factors for mild cognitive impairment $[1,2]$ and are associated the rate of cognitive decline in older adults [3]. The volume of WMH is associated with hypertension, especially at midlife [4]. Similarly, arterial stiffness at midlife, which often develops prior to hypertension [5], predicts future WMH volume [6]. Early changes in arterial structure of the central elastic arteries affect aortic hemodynamics prior to clinical changes in brachial BP [7]. Unlike diastolic $\mathrm{BP}$, which is similar when measured centrally (aortic) or peripherally (brachial), systolic BP is dependent on the location in the arterial tree where it is measured [7]. These differences in systolic BP are due to pulse pressure amplification and may have an impact on risk stratification [8]. In fact, some evidence suggests that treatment strategies to mitigate cardiovascular risk should be based on aortic hemodynamics [8, 9]. Thus, it is possible that, even though brachial systolic BP may be within the "normative" range, central or aortic hemodynamics may be associated with vascular brain injury as early as midlife.

Women are at elevated risk for developing hypertension after menopause [10]. However, in postmenopausal women without clinically defined hypertension, the volume of WMH as a fraction of total white matter volume associated with blood-borne markers of endothelial disruption, such as the thrombogenic microvesicles [11] suggesting that changes in WMH volume or fraction precede overt elevations in brachial BP. As there is inadequate examination of potential sex differences in the effects of hypertension and hormonal status on brain structure, this study sought to determine if aortic hemodynamics and arterial stiffness were associated with $\mathrm{WMH}$ in a cohort of postmenopausal women.

\section{Methods}

\section{Participants}

Women ( $n=74$ ) who had previously participated in the Kronos Early Estrogen Prevention Study (KEEPS) at Mayo Clinic were invited to participate in the present study. For participation in KEEPS, women were between 6 months and 3 years past their last menses and without prior cardiovascular events. Menopausal status was confirmed by $17 \beta$ estradiol and follicular stimulating hormone levels. Full inclusion criteria for KEEPS are reported elsewhere $[11,12]$. For the current study, women were recruited 3 years after their exit from the KEEPS study. The current study was to evaluate hemodynamic factors in these postmenopausal women in relationship to the fractional volume of WMH. Inclusion criteria were: (1) body mass index $(\mathrm{BMI})<35 \mathrm{~kg} / \mathrm{m}^{2}$; (2) no history of cardiovascular disease; (3) no diagnosis of diabetes; (4) no prescribed use of antihypertensive medication; (5) systolic brachial BP $<150 \mathrm{mmHg}$ and diastolic BP $<95 \mathrm{mmHg}$ during both study visits; (6) free of contraindications for MRI for safety such as an MRI-incompatible implant or claustrophobia; and (7) no neurologic diseases present that would have an impact on MRI findings such as multiple sclerosis, brain tumors, or epilepsy. All women underwent a neuropsychological assessment and their scores were within ageadjusted normative ranges [12]. All participants were nonsmokers.

\section{Standard protocol approvals, registrations and patient consents}

This study was approved by the Mayo Clinic Institutional Review Board. All participants gave written informed consent.

\section{Hemodynamic measurements}

Participants arrived to the Clinical Research Unit at Mayo Clinic between 11:00 and 13:30 after a 4-h fast and $24 \mathrm{~h}$ without caffeine, alcohol, and or exercise. Venous blood was collected for analysis of cardiometabolic risk factors (Table 1). Women rested in the supine position [13] and brachial BP was measured by the cuff method 3 times, each separated by $2 \mathrm{~min}$. Because normotensive individuals sometimes demonstrate higher than normal BP during laboratory study visits, women were excluded if their brachial BP was $>150 / 95 \mathrm{mmHg}$ or if they were using antihypertensive medications. After supine brachial BP measurements, aortic hemodynamics measurements were measured in the supine position. The radial arterial waveforms were calibrated from the supine brachial BP measurements taken immediately prior by an automated oscillometric device (Cardiocap/5, Datex-Ohmeda, Louisville, CO, USA). High-fidelity radial artery pressure waveforms were recorded by applanation tonometry of the radial pulse in the right wrist using a pencil type micromanometer (Millar Instruments, Houston, TX, USA). Multiple trials of sequential radial pulse waveforms were recorded over a 10-s period for each woman. Three to five trials of the radial pulse obtained in rapid succession were 
Table 1 Demographic and clinical characteristics

\begin{tabular}{ll}
\hline Variables & Median (IQR) \\
\hline Number of participants & 53 \\
Age (years) & $60(59,61)$ \\
Education, no. (\%) & \\
$\quad$ High school diploma & $3(6)$ \\
College graduate & $32(64)$ \\
Some graduate or professional & $2(4)$ \\
Graduate or professional degree & $12(24)$ \\
Time past menopause, months & $102(96,111)$ \\
Body mass index (kg/m $\left.{ }^{2}\right)$ & $27(23,31)$ \\
Waist circumference $(\mathrm{cm})$ & $87(79,96)$ \\
Total cholesterol $(\mathrm{mg} / \mathrm{dL})$ & $204(190,226)$ \\
Low-density lipoprotein $(\mathrm{mg} / \mathrm{dL})$ & $120(104,136)$ \\
High-density lipoprotein $(\mathrm{mg} / \mathrm{dL})$ & $64(57,75)$ \\
Triglycerides $(\mathrm{mg} / \mathrm{dL})$ & $88(72,109)$ \\
Fasting glucose $(\mathrm{mg} / \mathrm{dL})$ & $93(89,98)$ \\
hsCRP (pg/mL) & $1.2(0.5,2.6)$ \\
Estradiol (pg/mL) & $5(3,10)$ \\
Testosterone (ng/dL) & $15(11,21)$ \\
WMH fraction $(\%)$ & $0.0032(0.0020,0.0047)$ \\
\hline
\end{tabular}

hsCRP C-reactive protein, $W M H$ white matter hyperintensities

averaged for each participant [14]. A generalized transfer function (Sphygmocor, Atcor Medical, Sydney Australia) to correct for upper limb pressure amplification was used to generate the corresponding aortic pressure waveform and central BP. The generalized transfer function has been validated using both intra-arterially [15] and non-invasively obtained radial pressure waves [16].

The central aortic pressure waveform is composed of a forward traveling wave, generated by left ventricular ejection and a reflected wave that is returning to the ascending aorta from the periphery (Fig. 1). Pulse wave analysis of the aortic pressure waveform provided the following variables of interest: (1) central aortic BP; (2) augmented pressure (AP), the amplitude of the reflected wave which is defined as the difference between the first (forward wave) and second systolic shoulder of the aortic systolic BP; (3) AIx, augmentation index, the reflected wave amplitude divided by pulse pressure expressed as a percentage and adjusted for a heart rate of $75 \mathrm{bpm}$; (3) round trip travel time (Aortic $T_{\mathrm{R}}$ ) of the forward traveling wave from the ascending aorta to the major reflection site and back is measured from the beginning of the upstroke of the pressure wave to the foot of the reflected wave (inflection point); and (4) wasted left ventricular energy (LV wasted energy), which is the component of extra myocardial oxygen requirement due to early systolic wave reflection. LV wasted energy can be estimated as $1.333 *(\pi /$ $4)^{*}\left(\right.$ augmented pressure $* \Delta t_{\mathrm{r}}$ ), where 1.333 is the conversion factor and $\Delta t_{\mathrm{r}}$ is the time from the inflection point to the dicrotic notch (systolic duration of the reflected wave). Only high-quality recordings, defined as an in-device quality index of over $80 \%$ (derived from an algorithm including average pulse height variation, diastolic variation, and the maximum rate of rise of the peripheral waveform), were accepted for analysis. In general, 3-5 measurements were performed to obtain an acceptable quality index.

\section{Magnetic resonance imaging}

MRI was performed on a single 1.5-tesla system with an 8-channel phased-array head coil (GE Healthcare, Milwaukee, WI, USA) within 6 weeks of the hemodynamic measurements. Fluid-attenuated inversion recovery (FLAIR) MRI of the whole head was performed to quantify WMH volume. A 3-dimensional magnetization-prepared rapid acquisition gradient echo (MPRAGE) sequence was used for the segmentation of WM. All MRIs underwent preprocessing corrections for gradient nonlinearity and intensity nonuniformity.

WMH volumes were derived from a semi-automated segmentation of FLAIR images as detailed in previously published work [11]. Briefly, All MPRAGE and FLAIR images obtained during the same examination period were co-registered and segmented, and MPRAGE image was resampled in the FLAIR space. WMH on FLAIR images were segmented using an automated slice-based seed initialization and region-growing method, and the segmented WMH voxels were multiplied with the WMH mask. A trained image-analyst (S.Z.) inspected the segmented WMH mask overlaid on the FLAIR image. Every segmented slice was visually compared with the unprocessed FLAIR images and all false-positive WMH labels that resulted from artifacts were edited and excluded from the WMH mask [11]. To adjust for potential differences in WM volume among women, WMH fraction was calculated as $\mathrm{WMH}$ volume/total $\mathrm{WM}$ volume to determine the fraction of WM at risk.

\section{Data analysis and statistics}

A paired $t$ test was performed to determine if aortic and brachial systolic BP values were statistically different. In all analyses, WMH fraction was log-transformed to improve normality and reduce skewness. To assess the relationship between WMH fraction and aortic hemodynamic measures, we used linear regression models predicting $\log$ (WMH fraction) with each aortic hemodynamic measure taken one at a time while adjusting for age. The $t$ test and regression analyses were performed using statistical analysis software $\mathrm{R}$ version 3.1.1. 
Fig. 1 Typical applanation tonometry-derived ascending aortic pressure waveform with pulse wave analysis components including aortic systolic pressure; aortic diastolic pressure; inflection point where incident and reflected waves merge; or round trip travel time of reflected pressure wave to peripheral reflecting sites and back to heart; and AIx or augmentation index, the ratio of augmented pressure to pulse pressure

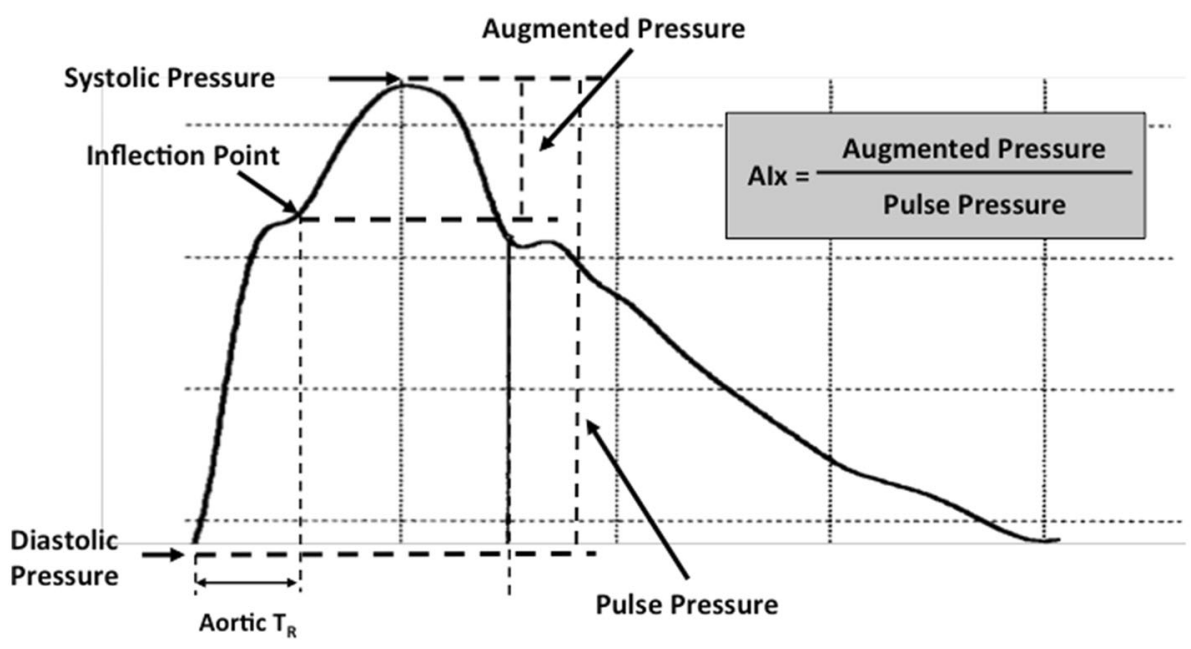

\section{Results}

\section{Participant characteristics}

Of the 74 women who agreed to participate in this study, twenty-one women were excluded from analysis due to a BMI $>35 \mathrm{~kg} / \mathrm{m}^{2}(n=8)$, presence of uncontrolled hypertension $(n=4)$, use of anti-hypertensive medication $(n=8)$, and feeling claustrophobic in the MRI scanner $(n=1)$. The demographic and clinical characteristics of the 53 women meeting inclusion criteria are shown in Table 1.

\section{Hemodynamic variables}

Median brachial and aortic systolic and diastolic pressures, IQR values for augmented pressure, AIx (adjusted for heart rate), Aortic $T_{\mathrm{R}}$ and LV wasted energy are listed in Table 2.

\section{Associations between WMH and aortic hemodynamic characteristics}

WMH fraction was positively associated with aortic systolic BP and AIx, and inversely associated with Aortic $T_{\mathrm{R}}$ (Table 3; Fig. 2). There was no association between WMH fraction and aortic diastolic BP, augmented pressure, or LV wasted energy ( $p>0.05$ for all; Table 3). Eliminating the effect of potential outliers (highest and lowest WMH fraction) did not change the results or statistical significance (data not shown).

\section{Associations between WMH and brachial BP}

The median brachial systolic BP was significantly greater than the median aortic systolic BP $(p<0.0001$; Table 2$)$; but median aortic diastolic BP did not differ from brachial diastolic BP $(p>0.05)$. There was a non-significant trend
Table 2 Brachial and aortic hemodynamics

\begin{tabular}{ll}
\hline Variables & Median (IQR) \\
\hline Brachial SBP (mmHg) & $123(115,127)$ \\
Brachial DBP (mmHg) & $73(69,79)$ \\
Brachial PP (mmHg) & $38(34,42)$ \\
Aortic SBP (mmHg) & $112(104,119)$ \\
Aortic DBP (mmHg) & $74(69,78)$ \\
Augmented pressure (mmHg) & $13(10,16)$ \\
AIx $(\%)$ & $27(23,31)$ \\
Aortic $T_{\mathrm{R}}(\mathrm{ms})$ & $141(133,146)$ \\
LV wasted energy (dynes $\left.\mathrm{cm}^{2} \mathrm{~s}\right)$ & $2.8(2.2,3.4)$ \\
\hline
\end{tabular}

$\mathrm{LV}$ wasted energy is in thousands

AIx aortic augmentation index at a heart rate of $75 \mathrm{bpm}$, Aortic $T_{R}$ round trip travel time, an indirect measure of aortic pulse wave velocity, $D B P$ diastolic blood pressure, $L V$ left ventricle, $P P$ pulse pressure, $S B P$ systolic blood pressure

for an association between WMH fraction and brachial systolic BP ( $p=0.08$, Table 3$)$. The age-adjusted association between WMH and brachial diastolic BP and brachial $\mathrm{PP}$ did not reach significance $(p=0.11, p=0.27$, respectively; Table 3).

\section{Association of WMH with other confounders}

WMH fraction did not associate with BMI or with MAP. Adjusting for height did not change the association between WMH fraction and aortic variables (data not shown).

\section{Discussion}

This study demonstrated that WMH fraction associated with aortic hemodynamic characteristics in postmenopausal women who were not using antihypertensive 
Table 3 Regression model estimates (SE) between log (WMH fraction) and hemodynamic variables adjusted for age

\begin{tabular}{llcc}
\hline Variable & Regression coefficient (SE) & $\begin{array}{c}\text { \% change in WMH fraction } \\
\text { for 1-unit increase in variable }\end{array}$ \\
\hline Brachial systolic BP (mmHg) & $0.018(0.01)$ & 1.8 & 0.08 \\
Brachial diastolic BP (mmHg) & $0.024(0.015)$ & 2.4 & 0.11 \\
Pulse pressure (mmHg) & $0.013(0.012)$ & 1.3 & 0.27 \\
Aortic systolic BP (mmHg) & $0.018(0.009)$ & 1.8 & 0.046 \\
Aortic diastolic BP (mmHg) & $0.028(0.015)$ & 3.2 & 0.07 \\
Augmented pressure (mmHg) & $0.032(0.019)$ & 2.5 & 0.10 \\
AIx (\%) @ 75 bpm & $0.025(0.011)$ & -1.5 & 0.04 \\
Aortic $T_{\mathrm{R}}$ (ms) & $-0.015(0.0073)$ & 10 & 0.04 \\
LV EW in thousands & $0.1(0.072)$ & 0.17 \\
\hline
\end{tabular}

Regression model beta estimates (standard error) between log (WMH fraction) and hemodynamic variables. All estimates were age-adjusted. Intercept estimates are not shown

AIx aortic augmentation index at a heart rate of $75 \mathrm{bpm}$, Aortic $T_{R}$ round trip travel time, an indirect measure of aortic pulse wave velocity, $B P$ blood pressure, $L V E W$ left ventricular wasted energy

Fig. 2 Scatterplot and regression line for aortic hemodynamic variables and white matter hyperintensity (WMH) fraction. The regression models include an additive ageeffect and the regression line is shown for the mean 60 years of age. AIx aortic augmentation index at a heart rate of $75 \mathrm{bpm}$, Aortic $T_{R}$ round trip travel time, an indirect measure of aortic pulse wave velocity, $D B P$ diastolic blood pressure, $S B P$ systolic blood pressure

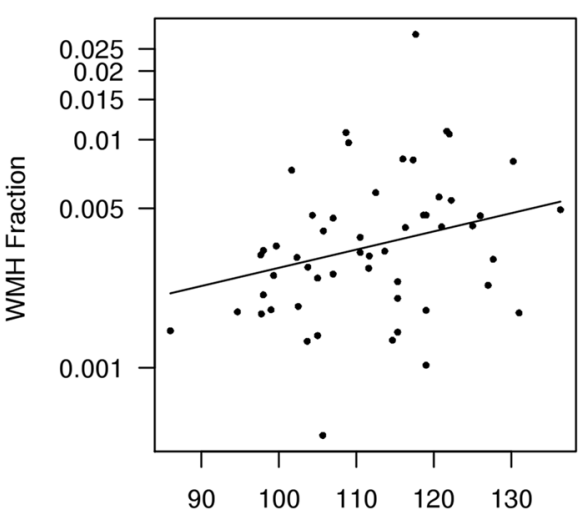

Aortic Systolic BP $(\mathrm{mmHg})$

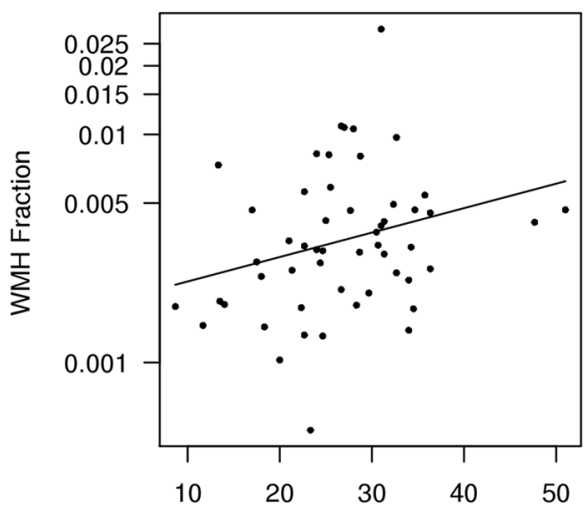

Alx (\%), $75 \mathrm{bpm}$

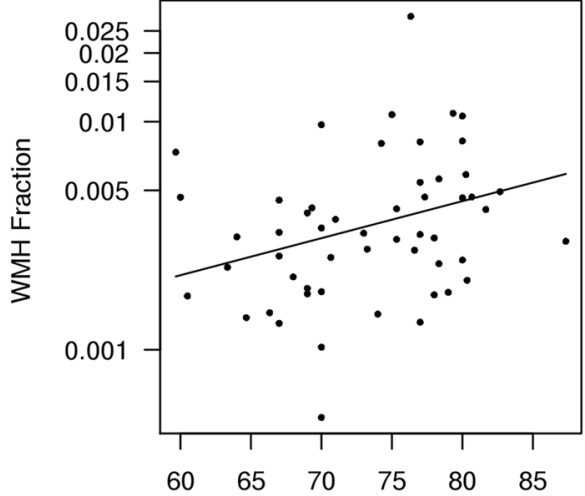

Aortic Diastolic BP $(\mathrm{mmHg})$

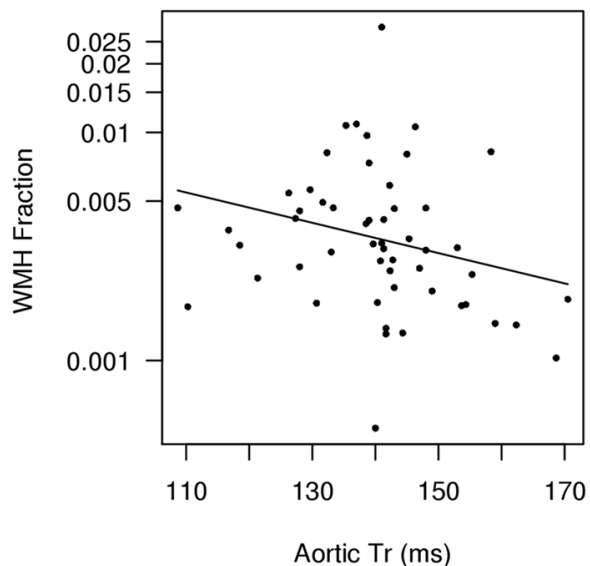

medications and who did not meet the clinical criteria for hypertension. Non-invasive pulse wave analysis has been used to detect changes in aortic characteristics for decades. The pressure waveform at the central aorta is important for assessing cardiovascular risk because it represents the work imposed on the left ventricle and central elastic arteries
[17]. A physiological increase in AIx reflects arterial stiffening, a change in downstream reflection sites, or elevated peripheral resistance. Similarly, a decrease in the time of reflection (Aortic $T_{\mathrm{R}}$ ) characterizes an increase in arterial stiffness. Unfavorable increases in aortic BP and AIx are associated with cardiovascular diseases such as 
hypertension, stroke, and coronary heart disease [18]. The present results expand these observations to show that the aortic parameters are also related to WMH burden in women who do not meet the current clinical definition for hypertension based on brachial arterial blood pressure. Therefore, assessing aortic hemodynamics and arterial stiffness in postmenopausal women with systemic brachial $\mathrm{BP}$ within what is considered pre-hypertensive ranges may be important in identifying risk for $\mathrm{WMH}$, and thus enable targeted prevention strategies.

A higher volume of WMH is associated with cardiovascular disease and risk factors such as age, myocardial infarction, hypertension, and atherosclerosis [19, 20]. During the transition to and after menopause, the risk of developing hypertension, ischemic heart disease, and stroke increases in women [21]. The decreases in ovarian hormones, in particular the loss of estrogen, during the transition to menopause may accelerate large artery stiffness, which can be detected in individuals with normal brachial BP values [5, 7, 22]. Indeed, sex differences in blood pressure parameters observed between young women and men [23], are not observed when postmenopausal women are compared to age-matched men [23, 24]. Therefore, aortic arterial hemodynamics may be a more sensitive measure of evaluating elevated risk for both cardiovascular and cerebrovascular disease than brachial BP.

The central elastic arteries are capable of transmitting pulsatile flow and cushion this pulsatility to smooth laminar flow in the peripheral arteries and microvessels. However, due to the short distance between the central elastic arteries and cerebral vasculature, the brain may be exposed to high pulsatile flow if this cushioning function is lost. A slight increase in arterial stiffness disrupts the ability of the elastic arteries to dampen the high pulsatility, so this can be transmitted to the cerebral vasculature. Whereas peripheral arteries are often protected from damage due to pulsatility because of downstream vasoconstriction, the brain is a high flow system with overall low impedance. The combination of the short distance between the central elastic arteries and the brain, as well as the high blood flow needs of the tissue, makes the brain particularly susceptible to damage from arterial stiffness [25]. Therefore, a small change in aortic hemodynamics or arterial stiffness can have a substantial impact on the microvasculature in the brain [25-27]. Indeed, in the present study, AIx and Aortic $T_{\mathrm{R}}$ measurements correlated with WMH fraction an observation that is consistent with the idea that pulse wave analysis provides a comprehensive assessment of the vascular stress placed on the cerebral circulation. The North American Artery Society recommends that pulse wave analysis be incorporated in guiding BP treatments in pre-hypertensive adults [27]. In addition, a recent study concluded that arterial stiffness predicted cognitive decline in healthy adults better than brachial BP [28]. Our results suggest that managing arterial stiffness and aortic BP, may be useful to reduce cardiovascular risk, and to reducing $\mathrm{WMH}$ burden that is associated with cognitive decline.

Previous studies in men and women have shown a positive correlation between aortic pulse wave velocity, a measure of arterial stiffness, and WMH volume. For example, pulse wave velocity associated with WMH in hypertensive adults [29], patients with recent minor stroke or transient ischemic attack [30], and patients without stroke or dementia in the Framingham Offspring Study [31]. A recent study demonstrated that even in young adults (age 30-45 years), aortic pulse wave velocity was associated with WMH volume [32]. Furthermore, aortic pulse wave velocity is associated with the WMH load measured after 10 years, suggesting that early increases in pulse wave velocity may precede WMH development 10 years later [6]. Additional follow-up of the women in our study is needed to better establish the clinical relevance between these hemodynamic measures, brain structure and cognition.

The main limitation of the present study is that the age range of the participants was narrow and the women had low risk cardiometabolic profiles. In addition, because this cohort consisted of white postmenopausal women, these findings cannot be generalized to postmenopausal women of different ethnic backgrounds, premenopausal women or men. Another limitation is that with this small sample, there was insufficient statistical power to adjust for all potential confounding variables. Despite these limitations, these findings suggest that even in postmenopausal women without clinically defined hypertension, higher aortic systolic BP, greater wave augmentation, and faster Aortic $T_{\mathrm{R}}$ are associated with greater WMH fraction. Because increases in aortic hemodynamics and arterial stiffness often precede changes in brachial BP measurements [5, 7], these results suggest that assessing aortic hemodynamics may identify normotensive women at increased risk for $\mathrm{WMH}$ and guide interventions to reduce development of WMH. Longitudinal evaluation of the participants is critical for determining the influence of WMH on cognitive outcomes.

Acknowledgements The authors thank Kim Jensen for consenting and scheduling of the participants.

Study funding This work was provided by National Institute of Health grants P50 AG044170 (LTS, VMM, MJJ, and KK) HL118154 (JNB), HL83947 (MJJ). Support for laboratory tests was provided, in part, by UL1 TR000135 (National Center for Advancing Translational Sciences (NCATS), a component of the National Institutes of Health (NIH). Its contents are solely the responsibility of the authors and do not necessarily represent the official view of $\mathrm{NIH}$ ), the Department of Surgery, Mayo Clinic and the Mayo Clinic Foundation. 
Author contributions Dr. Barnes: design or conceptualization of the study, data collection, analysis and interpretation of the data, drafting the manuscript; Ms. Harvey: data collection, analysis or interpretation of the data, revising the manuscript; Ms. Zuk: analysis or interpretation of the data, revising the manuscript; Ms. Lundt: analysis or interpretation of the data, revising the manuscript; Mr. Lesnick: analysis or interpretation of the data, revising the manuscript; Dr. Gunter: analysis or interpretation of the data, revising the manuscript; Mr. Senjem: analysis or interpretation of the data, revising the manuscript; Dr. Shuster: analysis or interpretation of the data, revising the manuscript; Dr. Miller: design or conceptualization of the study, data collection, analysis or interpretation of the data, revising the manuscript, study funding; Dr. Jack: analysis or interpretation of the data, revising the manuscript; Dr. Joyner: design or conceptualization of the study, data collection, revising the manuscript, study funding; Dr. Kantarci: design or conceptualization of the study, data collection, analysis and interpretation of the data, drafting the manuscript, study funding.

\section{Compliance with ethical standards}

Conflicts of interest Dr. Barnes is funded by NIH HL118154. Ms. Harvey, Ms. Zuk, Ms. Lundt, Mr. Lesnick, Dr. Gunter, Mr. Senjem do not report any disclosures. Dr. Shuster is funded by the NIH P50 AG44170. Dr. Miller is funded by the NIH P50 AG44170. Dr. Jack has provided consulting services for Eli Lilly Co. He receives research funding from the National Institutes of Health ((R01 AG011378, U01 HL096917, U01 AG024904, RO1 AG041851, R01 AG037551, R01 AG043392, U01 AG006786)) and the Alexander Family Alzheimer's Disease Research Professorship of the Mayo Foundation. Dr. Joyner is funded by NIH HL83947. Dr. Kantarci serves on the data safety monitoring board for Pfizer Inc., Takeda Global Research \& Development Center, Inc.; and she is funded by the NIH [R01AG040042 (PI), R21 NS066147 (PI), Mayo Clinic Alzheimer's Disease Research Center/Project 1 P50 AG16574/P1 (PI), P50 AG44170/Project 2 (PI) and R01 AG11378 (Co-I)].

Open Access This article is distributed under the terms of the Creative Commons Attribution 4.0 International License (http://crea tivecommons.org/licenses/by/4.0/), which permits unrestricted use, distribution, and reproduction in any medium, provided you give appropriate credit to the original author(s) and the source, provide a link to the Creative Commons license, and indicate if changes were made.

\section{References}

1. Kantarci K, Petersen RC, Przybelski SA, Weigand SD, Shiung MM, Whitwell JL, Negash S, Ivnik RJ, Boeve BF, Knopman DS, Smith GE, Jack CR Jr (2008) Hippocampal volumes, proton magnetic resonance spectroscopy metabolites, and cerebrovascular disease in mild cognitive impairment subtypes. Arch Neurol 65(12):1621-1628. doi:10.1001/archneur.65.12.1621

2. DeCarli C, Miller BL, Swan GE, Reed T, Wolf PA, Carmelli D (2001) Cerebrovascular and brain morphologic correlates of mild cognitive impairment in the National Heart, Lung, and Blood Institute Twin Study. Arch Neurol 58(4):643-647

3. Silbert LC, Nelson C, Howieson DB, Moore MM, Kaye JA (2008) Impact of white matter hyperintensity volume progression on rate of cognitive and motor decline. Neurology 71(2):108-113. doi:10.1212/01.wnl.0000316799.86917.37

4. Swan GE, DeCarli C, Miller BL, Reed T, Wolf PA, Jack LM, Carmelli D (1998) Association of midlife blood pressure to late- life cognitive decline and brain morphology. Neurology 51(4):986-993

5. Liao D, Arnett DK, Tyroler HA, Riley WA, Chambless LE, Szklo M, Heiss G (1999) Arterial stiffness and the development of hypertension. The ARIC study. Hypertension 34(2):201-206

6. Rosano C, Watson N, Chang Y, Newman AB, Aizenstein HJ, Du Y, Venkatraman V, Harris TB, Barinas-Mitchell E, Sutton-Tyrrell K (2013) Aortic pulse wave velocity predicts focal white matter hyperintensities in a biracial cohort of older adults. Hypertension 61(1):160-165. doi:10.1161/HYPERTENSIO NAHA.112.198069

7. Nichols WW, O'Rourke MF (2005) McDonalds's blood flow in arteries: theoretical, experimental and clinical principles, 5th edn. Hodder Arnold Publishing, London

8. Kollias A, Lagou S, Zeniodi ME, Boubouchairopoulou N, Stergiou GS (2016) Association of central versus brachial blood pressure with target-organ damage: systematic review and metaanalysis. Hypertension 67(1):183-190. doi:10.1161/HYPERTEN SIONAHA.115.06066

9. McEniery CM, Cockcroft JR, Roman MJ, Franklin SS, Wilkinson IB (2014) Central blood pressure: current evidence and clinical importance. Eur Heart J 35(26):1719-1725. doi:10.1093/eur heartj/eht565

10. Mosca L, Benjamin EJ, Berra K, Bezanson JL, Dolor RJ, LloydJones DM, Newby LK, Pina IL, Roger VL, Shaw LJ, Zhao D, Beckie TM, Bushnell C, D'Armiento J, Kris-Etherton PM, Fang J, Ganiats TG, Gomes AS, Gracia CR, Haan CK, Jackson EA, Judelson DR, Kelepouris E, Lavie CJ, Moore A, Nussmeier NA, Ofili E, Oparil S, Ouyang P, Pinn VW, Sherif K, Smith SC Jr, Sopko G, Chandra-Strobos N, Urbina EM, Vaccarino V, Wenger NK (2011) Effectiveness-based guidelines for the prevention of cardiovascular disease in women-2011 update: a guideline from the american heart association. Circulation 123(11):1243-1262. doi:10.1161/CIR.0b013e31820faaf8

11. Raz L, Jayachandran M, Tosakulwong N, Lesnick TG, Wille SM, Murphy MC, Senjem ML, Gunter JL, Vemuri P, Jack CR Jr, Miller VM, Kantarci K (2013) Thrombogenic microvesicles and white matter hyperintensities in postmenopausal women. Neurology 80(10):911-918. doi:10.1212/WNL.0b013e3182840c9f

12. Kantarci K, Lowe VJ, Lesnick TG, Tosakulwong N, Bailey KR, Fields JA, Shuster LT, Zuk SM, Senjem ML, Mielke MM, Gleason C, Jack CR, Rocca WA, Miller VM (2016) Early postmenopausal transdermal 17beta-estradiol therapy and amyloidbeta deposition. J Alzheimers Dis. doi:10.3233/JAD-160258

13. Netea RT, Lenders JW, Smits P, Thien T (2003) Both body and arm position significantly influence blood pressure measurement. J Hum Hypertens 17(7):459-462. doi:10.1038/sj.jhh.1001573

14. Barnes JN, Casey DP, Hines CN, Nicholson WT, Joyner MJ (2012) Cyclooxygenase inhibition augments central blood pressure and aortic wave reflection in aging humans. Am J Physiol Heart Circ Physiol 302(12):H2629-H2634. doi:10.1152/ajpheart.00032.2012

15. Chen CH, Nevo E, Fetics B, Pak PH, Yin FC, Maughan WL, Kass DA (1997) Estimation of central aortic pressure waveform by mathematical transformation of radial tonometry pressure. Validation of generalized transfer function. Circulation 95(7):1827-1836

16. Gallagher D, Adji A, O'Rourke MF (2004) Validation of the transfer function technique for generating central from peripheral upper limb pressure waveform. Am J Hypertens 17(11 Pt 1):1059-1067. doi:10.1016/j.amjhyper.2004.05.027

17. Laurent S, Cockcroft J, Van Bortel L, Boutouyrie P, Giannattasio C, Hayoz D, Pannier B, Vlachopoulos C, Wilkinson I, StruijkerBoudier H, European Network for Non-invasive Investigation of Large A (2006) Expert consensus document on arterial stiffness: methodological issues and clinical applications. Eur Heart $\mathbf{J}$ 27(21):2588-2605. doi:10.1093/eurheartj/ehl254 
18. Weber T, Auer J, O’Rourke MF, Kvas E, Lassnig E, Berent R, Eber B (2004) Arterial stiffness, wave reflections, and the risk of coronary artery disease. Circulation 109(2):184-189. doi:10. 1161/01.CIR.0000105767.94169.E3

19. Breteler MM, van Swieten JC, Bots ML, Grobbee DE, Claus JJ, van den Hout JH, van Harskamp F, Tanghe HL, de Jong PT, van Gijn J, Hofman A (1994) Cerebral white matter lesions, vascular risk factors, and cognitive function in a population-based study: the Rotterdam Study. Neurology 44(7):1246-1252

20. Dufouil C, de Kersaint-Gilly A, Besancon V, Levy C, Auffray E, Brunnereau L, Alperovitch A, Tzourio C (2001) Longitudinal study of blood pressure and white matter hyperintensities: the EVA MRI cohort. Neurology 56(7):921-926

21. Rocca WA, Grossardt BR, Miller VM, Shuster LT, Brown RD Jr (2012) Premature menopause or early menopause and risk of ischemic stroke. Menopause 19(3):272-277. doi:10.1097/gme. 0b013e31822a9937

22. Rajkumar C, Kingwell BA, Cameron JD, Waddell T, Mehra R, Christophidis N, Komesaroff PA, McGrath B, Jennings GL, Sudhir K, Dart AM (1997) Hormonal therapy increases arterial compliance in postmenopausal women. J Am Coll Cardiol 30(2):350-356

23. Waddell TK, Dart AM, Gatzka CD, Cameron JD, Kingwell BA (2001) Women exhibit a greater age-related increase in proximal aortic stiffness than men. J Hypertens 19(12):2205-2212

24. Berry KL, Cameron JD, Dart AM, Dewar EM, Gatzka CD, Jennings GL, Liang YL, Reid CM, Kingwell BA (2004) Largeartery stiffness contributes to the greater prevalence of systolic hypertension in elderly women. J Am Geriatr Soc 52(3):368-373

25. Mitchell GF (2008) Effects of central arterial aging on the structure and function of the peripheral vasculature: implications for end-organ damage. J Appl Physiol 105(5):1652-1660

26. O'Rourke MF, Safar ME (2005) Relationship between aortic stiffening and microvascular disease in brain and kidney: cause and logic of therapy. Hypertension 46(1):200-204. doi:10.1161/ 01.HYP.0000168052.00426.65

27. Townsend RR, Black HR, Chirinos JA, Feig PU, Ferdinand KC, Germain M, Rosendorff C, Steigerwalt SP, Stepanek JA (2015) Clinical use of pulse wave analysis: proceedings from a symposium sponsored by North American artery. J Clin Hypertens (Greenwich) 17(7):503-513. doi:10.1111/jch.12574

28. Hajjar I, Goldstein FC, Martin GS, Quyyumi AA (2016) Roles of arterial stiffness and blood pressure in hypertension-associated cognitive decline in healthy adults. Hypertension 67(1):171-175. doi:10.1161/HYPERTENSIONAHA.115.06277

29. Brandts A, van Elderen SG, Westenberg JJ, van der Grond J, van Buchem MA, Huisman MV, Kroft LJ, Tamsma JT, de Roos A (2009) Association of aortic arch pulse wave velocity with left ventricular mass and lacunar brain infarcts in hypertensive patients: assessment with MR imaging. Radiology 253(3):681-688. doi:10.1148/radiol.2533082264

30. Webb AJ, Simoni M, Mazzucco S, Kuker W, Schulz U, Rothwell PM (2012) Increased cerebral arterial pulsatility in patients with leukoaraiosis: arterial stiffness enhances transmission of aortic pulsatility. Stroke 43(10):2631-2636. doi:10.1161/STRO KEAHA.112.655837

31. Tsao CW, Seshadri S, Beiser AS, Westwood AJ, Decarli C, Au R, Himali JJ, Hamburg NM, Vita JA, Levy D, Larson MG, Benjamin EJ, Wolf PA, Vasan RS, Mitchell GF (2013) Relations of arterial stiffness and endothelial function to brain aging in the community. Neurology 81(11):984-991. doi:10.1212/WNL. 0b013e3182a43e1c

32. Pase MP, Himali JJ, Mitchell GF, Beiser A, Maillard P, Tsao C, Larson MG, DeCarli C, Vasan RS, Seshadri S (2016) Association of aortic stiffness with cognition and brain aging in young and middle-aged adults: the Framingham third generation cohort study. Hypertension. doi:10.1161/HYPERTENSIONAHA.115. 06610 